\title{
A Case of a Non-Radiographic Axial Spondyloarthritis in a 14-Year-Old Girl Presenting with Muscle Weakness and Hyperthyroidism
}

This article was published in the following Dove Press journal:

International Medical Case Reports Journal

\author{
Abdullah Elhosiny (D' \\ Renad Alshoubaki ${ }^{1}$ \\ Lama Sindi $\mathbb{D}^{2}$ \\ Sadeem Bukhari (iD ${ }^{2}$ \\ Hani Almoallim (D) ${ }^{1,2}$ \\ 'Department of Medicine, Dr. Soliman \\ Fakeeh Hospital, Jeddah, Saudi Arabia; \\ ${ }^{2}$ Department of Medicine, Faculty of \\ Medicine, Umm Alqura University, \\ Makkah, Saudi Arabia
}

\begin{abstract}
Juvenile spondyloarthritis (SpA) affects patients below the age of 16 and mainly presents with peripheral involvement. Many atypical clinical presentations were reported in the adult $\mathrm{SpA}$, but not in the juvenile group. We report a case of non-radiographic axial SpA in a 14-year-old girl presented atypically with muscle weakness and hyperthyroidism. She had sacroiliitis evident by magnetic resonance imaging (MRI) with negative HLA-B27. Muscle biopsy showed fasciitis. She responded well to adalimumab and thyroid functions returned to normal values.
\end{abstract}

Keywords: juvenile spondyloarthritis, non-radiographic spondyloarthritis, hyperthyroidism, muscle weakness, ankylosing spondylitis, spondyloarthritis

\section{Introduction}

Spondyloarthritis $(\mathrm{SpA})$ is a group of interrelated diseases with axial and peripheral inflammatory joint involvement along with many extra-articular manifestations. Juvenile SpA is a subgroup from the SpA family that affects patients less than 16 years of age. ${ }^{1}$

Clinical manifestation of SpA differs widely, juvenile SpA usually presents with peripheral joint involvement and enthesitis while axial involvement is not common and usually asymptomatic. Adult population on the contrary manifests as an inflammatory back pain. ${ }^{2}$ Additionally, SpA can present with different atypical presentations that include fever of unknown origin, ${ }^{3}$ thigh pain, anterior chest wall pain, polymyalgia rheumatica, ${ }^{4}$ and valvular heart disease. ${ }^{5}$

SpA has been associated with many different endocrinopathies including thyroid disorders (hyper and hypothyroidism, thyroid nodules, parenchymatous changes of the thyroid gland, and the presence of thyroid antibodies). ${ }^{6}$

We report here a young patient who was diagnosed accidentally with juvenile SpA after she presented initially with profound muscle weakness and hyperthyroidism.

\section{Case Report}

A 14-year-old female patient, presented to the emergency department complaining of progressive proximal muscle weakness for the past 2 months. She noticed difficulty in combing her hair and in standing from sitting position. Her symptoms then progressed over a period of 2 months till she became unable to walk without support, eventually, she became wheelchair bound.
Correspondence: Hani Almoallim Faculty of Medicine, Umm Alqura University (UQU)

Email hanialmoallim@gmail.com 
Upon further questioning, she described morning stiffiness that involved both small hand joints and lasted for about 30 mins. In addition, she reported dysphagia to both solid and liquids.

She had a poor appetite but denied a history of fever or weight loss. Systemic review was otherwise unremarkable. Her past medical history was significant for thyroiditis back in 2015, treated with supportive measures and resolved without the need for chronic medication use. Family history was significant for Prader Willi syndrome in her younger sister.

Patient was up to date on regard to her vaccination based on the Saudi Arabia: WHO and UNICEF estimates of immunization coverage: 2018 revision. ${ }^{7}$ No prior history of a vaccination that contained aluminum hydroxide nor other similar high-risk substances. And her diffuse muscle weakness was not at the site of her previous vaccinations. ${ }^{15}$

Physical examination upon presentation showed normal vital signs. Neurological examination revealed decreased power $4 / 5$ in the upper limbs bilaterally and symmetrically, and 3/5 in the lower limbs bilaterally. She had brisk reflexes more on the left side of her body. Her gait was normal but the patient was unable to stand from the wheel chair unaided. She was unable to make a fist or tuck position during left-hand examination with tenderness in the fourth and fifth proximal interphalangeal (PIP) and metacarpophalangeal (MCP) joints bilaterally. The remainder of her physical examination was unremarkable.

Patient was admitted and her laboratory investigations revealed the following: leukopenia, white blood cells of $2.96\left(4.5-13.5\left(10^{3} / \mu \mathrm{L}\right)\right)$, with microcytic hypochromic anemia, hemoglobin of $10.4(11.5-15.5(\mathrm{gm} / \mathrm{dl}))$, with high inflammatory marker, C-reactive protein of 18.8 (0.0-5.0 (mg/L), erythrocyte sedimentation rate $(109.2$ $(0.0-20.0(\mathrm{~mm} / \mathrm{hr})))$, creatinine kinase total $39(29.0$ $168.0(\mathrm{U} / \mathrm{L})$ ), thyroid function test and antibody: TSH: $0.017(0.35-4.94(\mu \mathrm{lU} / \mathrm{mL}))$, T3 (FREE): 4.53 (2.6-5.7 (pmol/l)), T4 (FREE): 20.9 (9.0-19.0 (pmol/l)), anti-thyroglobulin $3410(5-100(\mathrm{IU} / \mathrm{mL}))$. All autoimmune workup including rheumatoid factor, anti-citrullinated peptide antibodies, antineutrophil cytoplasmic antibodies were negative except antinuclear antibody of $27(<20)$. All viral serologies were negative as well. Renal function, liver enzymes and hormonal assays were all normal. Hyperkalemic paralysis was ruled out.

Thyroid ultrasound showed multinodular goiter consistent with thyroiditis. Thyroid scintigraphy was not done due to recently performed MRI. Endocrinology assessment suggested that this patients' weakness was less likely related to thyroiditis. Other endocrine causes of weakness like adrenal insufficiency and cushing's disease were ruled out. Neurology assessment again did not reveal any suggestive etiology like multiple sclerosis as her MRI brain and lumbosacral spine were normal. MRI cervical spine showed only small disc herniation at C6-C7.

An accidental finding of bilateral sacroiliitis was noted in her lumbosacral spine MRI. Rheumatology consultation was done at that time. Further history revealed chronic low back pain which was mild along with a history of small joints pain as outlined above. X-ray of her sacroiliac joints was normal but her sacroiliac joint MRI showed evidence of bilateral sacroiliitis (Figure 1). HLA-B27 was negative. MRI hip and pelvis were arranged to assess for the presence of myositis. This showed bilateral thigh intramuscular edema with an enhancement that involved the left thigh, the right vastus lateral, bilateral obturator internus and left gluteus maximus muscles. Myositis was suspected but CKtotal and electromyography (EMG) were both normal. After extensive discussion between different managing teams, a muscle biopsy was performed. However, the findings were available almost 3 weeks after the biopsy was taken due to logistical reasons.

The working diagnosis then as suggested by the rheumatology assessment was non-radiographic axial SpA with possible myositis. The patient was started initially on ibuprofen (NSAIDs) followed by prednisone which was given for 1 week duration for presumed thyroiditis.

Meanwhile, gastrografin swallow assessment for her dysphagia was normal. Endoscopy was done after discharge and revealed gastroesophageal reflux disease (GERD) with normal biopsy finding thus she was maintained on a proton pump inhibitor. She started to tolerate oral food better and her weight increased.

The patient started to show mild improvement during hospitalization and she was discharged to be followed up in rheumatology clinic.

During her follow-up at the rheumatology clinic, her symptom persisted with only minimal improvement from her baseline weakness. Muscle biopsy results came back from the left thigh and showed chronic macrophage/ inflammatory infiltration, mainly in the fascia and the peri fascial connective tissue (Figures 2 and 3). The most likely diagnosis based on this result was macrophage myofasciitis.

She was started on adalimumab after a negative result of TB-Quantefron and a normal chest $\mathrm{x}$-ray. She responded very well with good control of her symptoms. During her regular 


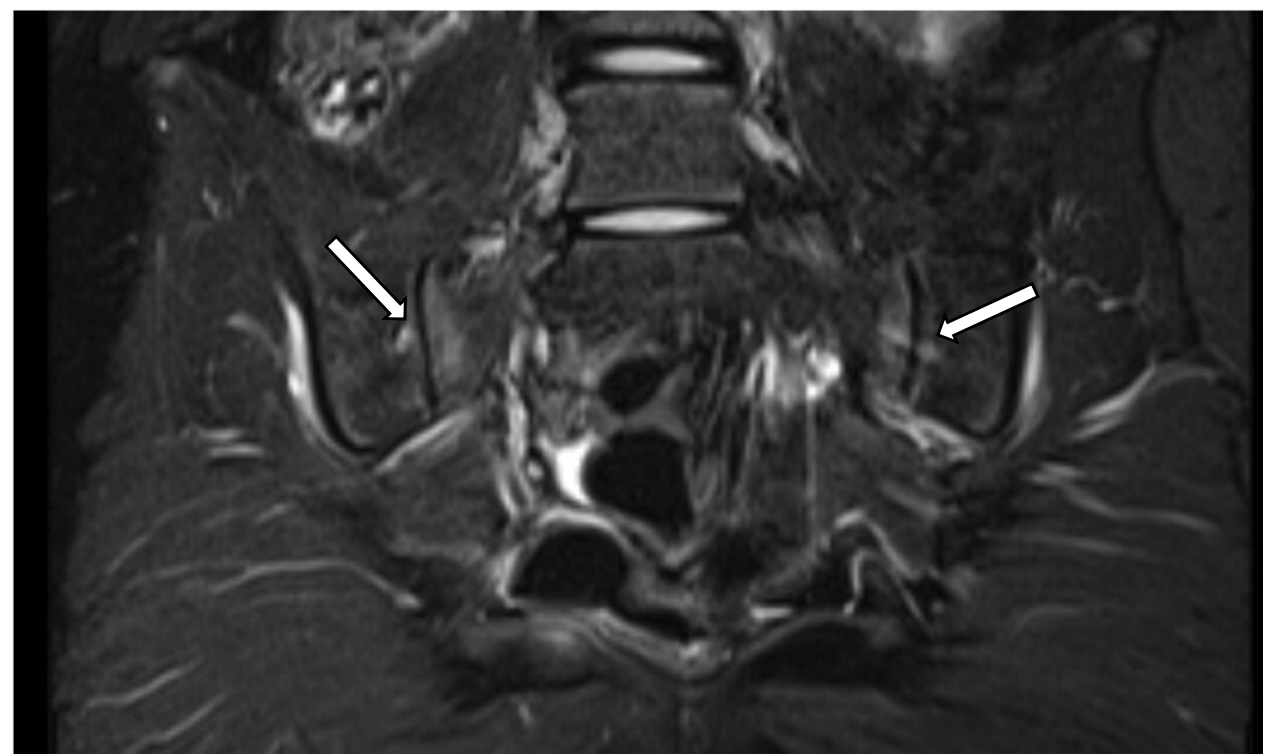

Figure I High-intensity signals involving both sacroiliac joints suggestive of bilateral sacroiliitis.

follow-up visits in the rheumatology clinic for up to 12 months post discharge her thyroid function improved back to normal on two separate settings. She remained symptoms free on adalimumab and have resumed her usual daily activities.

\section{Discussion}

We report here a case of juvenile non-radiographic SpA that was discovered accidentally. Our young female patient presented atypically with profound muscle weakness and hyperthyroidism. She did have musculoskeletal complaints

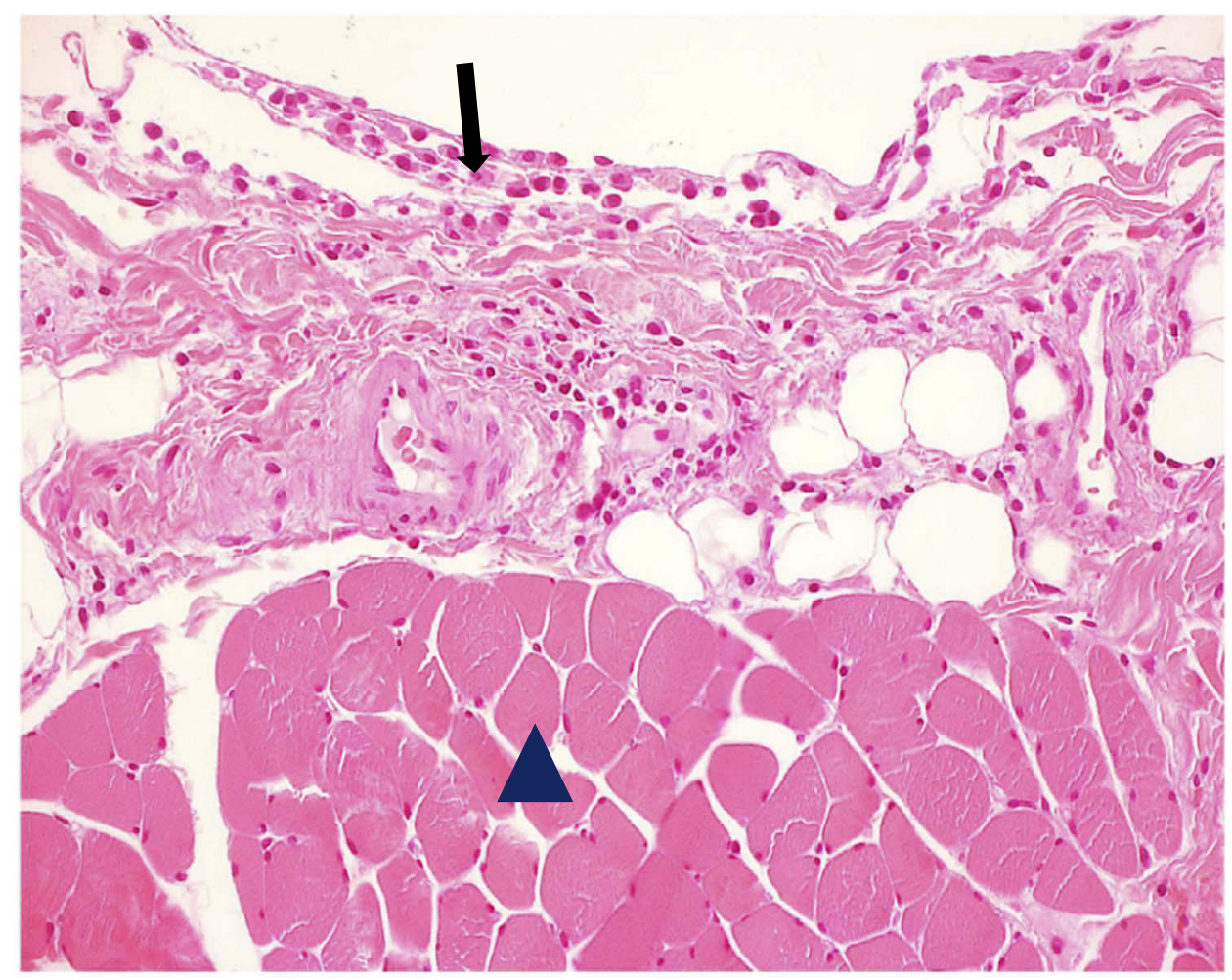

Figure 2 Muscle biopsy shows focal infiltration of the epimysium by large mononuclear cells of the monocyte and macrophage lineage (arrow), admixed with a small number of lymphocytes. Note that the muscle fibers remote from the inflammatory infiltrate are intact (arrowhead) (H\&Ex 100). 


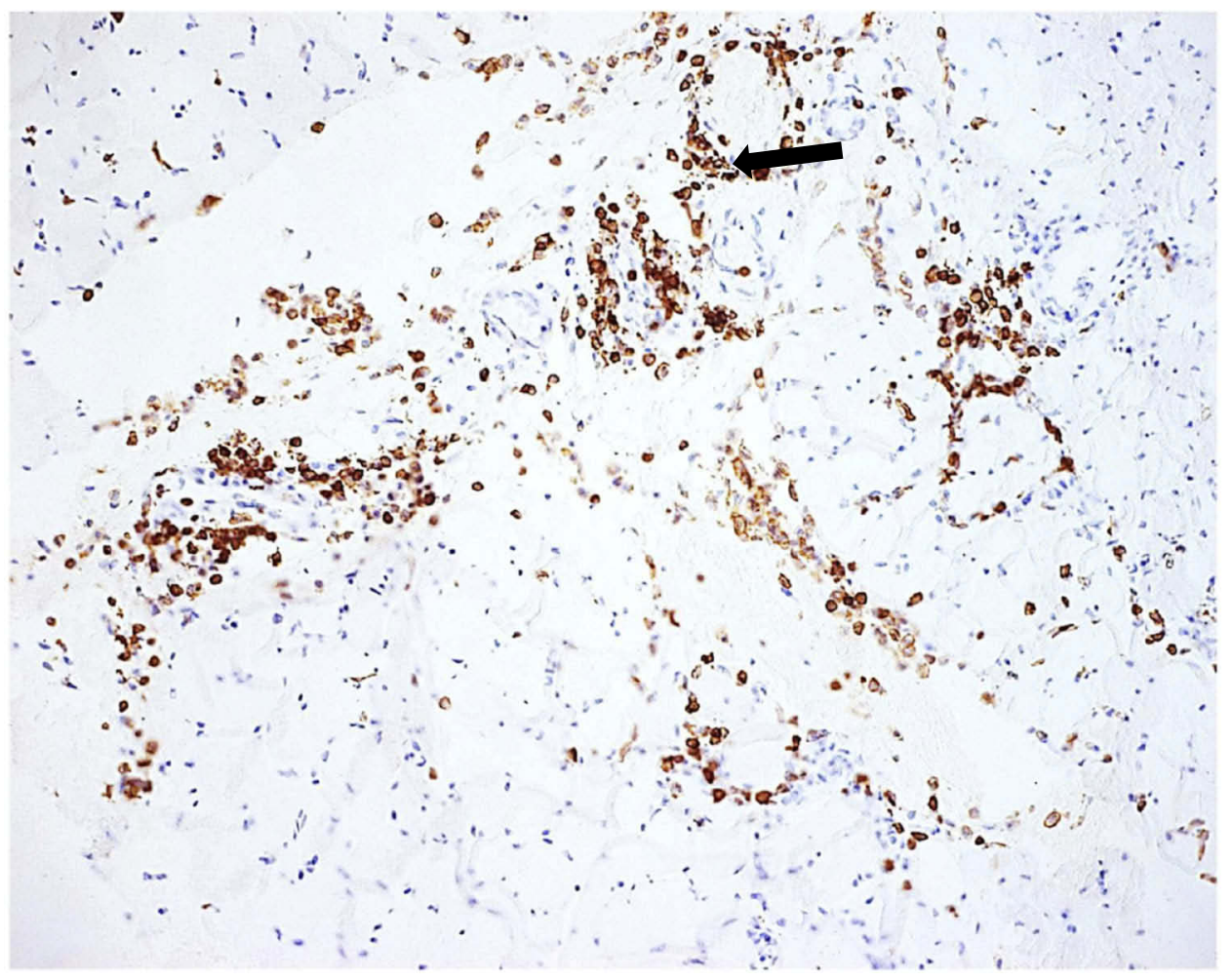

Figure 3 CD68-positive macrophages forming perivascular cuffs are present (arrow) (anti CD68×100).

that were properly over looked by her weakness. This reflects how it is difficult sometimes to diagnose rheumatological conditions in this young age group. Despite the abnormal MRI findings of thigh and pelvic muscles that suggested myositis, the muscle biopsy revealed only macrophage fasciitis. It is an unexpected finding and raise a question if this can be a form of enthesitis; the universal hallmark in SpA? This patient however responded remarkably to adalimumab.

Juvenile $\mathrm{SpA}$ is a rare type of SpA and affects children below the age of 16 where the youngest patient reported was a 6 years old boy. ${ }^{8}$ It presents typically with enthesitis and peripheral involvement. Axial sacroiliitis is not common among this group. Those children, most of the time are missed diagnosed with other differentials, especially when they present with an atypical clinical feature along with non-radiographic axial involvement like our young girl. The unusual atypical presentations were mainly reported among adult SpA. What was even more interesting in our patient is the presence of macrophage fasciitis which is extremely rare in axial SpA with almost no data in the literature, even in the adult population.

Another unusual feature here in our patient was the findings of hyperthyroidism and thyroid nodules. We may consider this as an initial rare atypical presentation of her non-radiographic axial $\mathrm{SpA}$. It is established that most of juvenile axial SpA are asymptomatic. Her chronic low back pain might have been overlooked due to profound fatigability and weakness. There is a mixture of data in the literature about the association between thyroid gland and axial SpA. Nonetheless, endocrinopathies and the field of rheumatology does possess connections as many organs are linked in a way or another with the immune system.

The prevalence of autoimmune thyroid disorders is much higher among SpA patients compared to the normal population, especially if the diseases activity is not controlled. ${ }^{9}$ More in depth, a study ${ }^{6}$ has shown many different forms of thyroid disorders seen among patient with $\mathrm{SpA}$ in which hypoechoic pattern on ultrasonography (US) was observed the most reaching up to $38 \%$. Other form of thyroid disorders included; the presence of thyroglobulin (TG) antibodies, thyroid peroxidase (TPO) antibodies, thyroid nodules, hyper and hypothyroidism. ${ }^{6}$ Almost all types of SpA had significantly higher prevalence of having one or more thyroid disorders except ankylosing spondylitis (AS) which had none at all; ${ }^{6}$ this however can be contributed to the small number of AS patients compared to other types of SpA. ${ }^{6}$ 
On another comparative study in AS patients, a higher prevalence of thyroid parenchymatous changes with a higher presence of thyroid antibodies was found among AS group compared to the healthy group, but no difference in the thyroid function as almost all of them were in the euthyroid state. ${ }^{10}$ What was more interesting here is that all the significant values were observed on males and almost no significant value was noted in the female group when compared to the healthy group. ${ }^{10}$

On the contrary, a retrospective cohort review of SpA patients found no significant value when looking for thyroid autoimmunity. ${ }^{1}$ A case-control study aimed to look for these thyroid disorders among patients with AS found that AS group had higher US thyroid hypoechogenicity of $30 \%$ and higher thyroid autoantibodies positivity of $13 \%$ compared to the healthy group. ${ }^{11}$ These conflicting findings in different reports from different places raise the concern about the validity of screening young patients with significant thyroid disorders for features of SpA.

It should be noted that the associations between thyroid disorders and SpA were studied among patients already diagnosed with SpA. On the other hand, our patient in this report presented with an initial picture of thyroid nodule and hyperthyroidism rather than the typical picture of axial SpA. This could support the association between the two disorders and it can consider thyroid disorders as an initial atypical presentation of Juvenile non-radiographic axial SpA. Clearly, more studies are needed to clarify all these issues.

Our patient responded remarkably well to adalimumab. This is consistent with adalimumab efficacy in non-radiographic SpA in the young age group. ${ }^{12,13}$ It is interesting to note that in a retrospective study that was done among AS patients who received anti-TNF therapy, less thyroid TPO antibodies were noted compared to those who did not. ${ }^{14}$ There was no other significant difference with regard to other thyroid disorders between the two groups.

Autoimmune diseases and endocrinopathies carry a strong relationship between each other with a wide variety of clinical presentations that are yet to be discovered. From the conflict of data along with the clinical findings in our young patient, thyroid disorder can be considered as an initial clinical presentation for juvenile axial $\mathrm{SpA}$ and not just a mere association. This will broaden up both the clinical approach of thyroid disorders along with the research opportunities in this field. Muscle weakness can be attributed to macrophage fasciitis. However, we cannot conclude if her macrophage myofasciitis improvement was related to the use of adalimumab or the resolution of her thyroid disorder. May we consider macrophage myofasciitis a form of enthesitis? Adalimumab was an effective treatment modality for this patient with non-radiographic axial SpA.

\section{Ethical Consent}

Written and informed consent was taken from the patients' father for the publications of the patients' information and her accompanying images. Institutional approval is not required to publish the case details.

\section{Acknowledgment}

This work was funded and supported by Alzaidi Chair of Research in Rheumatic Disease, Umm Alqura University, Makkah, Saudi Arabia.

\section{Disclosure}

The authors report no conflicts of interest in this work.

\section{References}

1. Pérez-Fernández OM, Mantilla RD, Cruz-Tapias P, RodriguezRodriguez A, Rojas-Villarraga A, Anaya J-M. Spondyloarthropathies in autoimmune diseases and vice versa. Autoimmune Dis. 2012;2012:736384.

2. Sieper J, Rudwaleit M, Khan MA, Braun J. Concepts and epidemiology of spondyloarthritis. Best Pract Res Clin Rheumatol. 2006;20 (3):401-417. doi:10.1016/j.berh.2006.02.001

3. Byun SJ, Bae WH, Jung SM, Lee S-W, Park Y-B, Song JJ. Fever as an initial manifestation of spondyloarthritis: A retrospective study. PLoS One. 2017;12(9):e0184323-e. doi:10.1371/journal.pone.0184 323

4. Hmamouchi I, Bahiri R, Hajjaj-Hassouni N. Clinical and radiological presentations of late-onset spondyloarthritis. ISRN Rheumatol. 2011;2011:840475. doi:10.5402/2011/840475

5. L, Sadek M, McDonald-Blumer H, Gladman DD, editors. Aortitis and spondyloarthritis - an unusual presentation: case report and review of the literature.Seminars in Arthritis and Rheumatism. 2010;39(6):510-514.

6. Peluso R, Lupoli GA, Del Puente A, et al. Prevalence of thyroid autoimmunity in patients with spondyloarthropathies. J Rheumatol. 2011;38(7):1371-1377. doi:10.3899/jrheum.101012

7. Health Organization. Saudi Arabia: WHO and UNICEF estimates of immunization coverage: 2018 revision. Available from:https://www. who.int/immunization/monitoring_surveillance/data/sau.pdf. Accessed February 17, 2020.

8. Hartman GH, Renaud DL, Sundaram M, Reed AM. Spondyloarthropathy presenting at a young age: case report and review. Skeletal Radiol. 2007;36(2):161-164. doi:10.1007/s00256006-0103-7

9. Chakravarty SD, Markenson JA. Rheumatic manifestations of endocrine disease. Curr Opin Rheumatol. 2013;25(1):37-43. doi:10.1097/ BOR.0b013e32835b4f3f

10. Emmungil H, Erdogan M, Kalfa M, et al. Autoimmune thyroid disease in ankylosing spondylitis. Clin Rheumatol. 2014;33(7):955961. doi:10.1007/s10067-013-2466-1 
11. Emmungil H, Erdogan M, Kalfa M, et al. The prevalence of autoimmune thyroid disfunction in ankylosing spondylitis. Am Coll Rheumatol. 2012;532:64.

12. Sulpice M, Deslandre CJ, Quartier P. Efficacy and safety of TNFalpha antagonist therapy in patients with juvenile spondyloarthropathies. Joint Bone Spine. 2009;76(1):24-27. doi:10.1016/j.jbspin.2008.03.008

13. Callhoff J, Sieper J, Weiss A, Zink A, Listing J. Efficacy of TNFalpha blockers in patients with ankylosing spondylitis and nonradiographic axial spondyloarthritis: a meta-analysis. Ann Rheum Dis. 2015;74(6):1241-1248. doi:10.1136/annrheumdis-2014-205322
14. Tarhan F, Orük G, Niflioğlu O, Ozer S. Thyroid involvement in ankylosing spondylitis and relationship of thyroid dysfunction with anti-TNF $\alpha$ treatment. Rheumatol Int. 2013;33(4):853-857. doi:10.1007/s00296-012-2438-9

15. Rinnenthal JL, Goebel -H-H, Preuße C, et al. Inflammatory myopathy with abundant macrophages (IMAM): the immunology revisited. Neuromuscul Disord. 2013. Elsevier.

\section{Publish your work in this journal}

The International Medical Case Reports Journal is an international, peer-reviewed open-access journal publishing original case reports from all medical specialties. Previously unpublished medical posters are also accepted relating to any area of clinical or preclinical science. Submissions should not normally exceed 2,000 words or 4 published pages including figures, diagrams and references. The manuscript management system is completely online and includes a very quick and fair peer-review system, which is all easy to use. Visit http://www.dovepress.com/testimonials.php to read real quotes from published authors. 